\title{
VALIDACIÓN DEL INVENTARIO DE ANSIEDAD Y FOBIA SOCIAL EN UNA MUESTRA DE ESTUDIANTES UNIVERSITARIOS MEXICANOS
}

VALIDATION OF THE SOCIAL PHOBIA AND ANXIETY INVENTORY IN A SAMPLE OF
MEXICAN UNIVERSITY STUDENTS

Recibido: 29 de Mayo del 2012 | Aceptado: 18 de Diciembre del 2012

\begin{abstract}
JOSÉ MORAL DE LA RUBIA ${ }_{1}$ CÉSAR JESÚS ANTONA CASAS 2 ; CIRILO HUMBERTO GARCÍA CÁRDENAS 3 (UNIVERSIDAD AUTÓNOMA DE NUEVO LEÓN, Nuevo León, México)
\end{abstract}

\begin{abstract}
RESUMEN
Este artículo tiene como objetivos estudiar la estructura factorial, consistencia interna, distribución, diferencias entre sexos, validez convergente y relación con la edad y deseabilidad social del Inventario de ansiedad y fobia social (Social Phobia and Anxiety Inventory [SPAI]). En una muestra de 695 estudiantes universitarios con cuotas equivalentes por sexos se aplicaron el SPAI, la Escala de ansiedad en la interacción social (Social Interaction Anxiety Scale [SIAS]), la Escala de autoverbalizaciones al hablar en público (Self-Statements during Public Speaking Scale [SSPS] y el Inventario balanceado de deseabilidad social al responder (Balanced Inventory of Desirable Responding [BIDR]). Reducido el SPAI a 41 ítems, el modelo estructural de un factor de fobia social (con cinco factores subordinados) correlacionado con un factor de agorafobia mostró un ajuste adecuado a los datos. Los valores de consistencia interna fueron altos, las distribuciones asimétricas positivas, las mujeres promediaron más alto que los hombres, las correlaciones fueron bajas con edad y el factor de manejo de la impresión del BIDR, y moderadas con SIAS y SSPS. Se sugiere el uso del SPAI-4I en México y su estudio en otros países hispanoparlantes.
\end{abstract}

PALABRAS CLAVE: Agorafobia; ansiedad social; deseabilidad social; fobia social.

\section{ABSTRACT}

The aims of this paper were to study the factorial structure, internal consistency, distribution, sex differences, convergent validity, and the relation to age and social desirability of the Social Phobia and Anxiety Inventory (SPAI). The SPAI, Social Interaction Anxiety Scale (SIAS), Self-statements Scale during Public Speaking (SSPS) and Balanced Inventory of Desirable Responding (BIDR), were administered to a sample of 695 college students with equivalent gender quotas. If the SPAI is reduced to 41 items, a structural model of one factor of social phobia (with five subordinate factors) correlated with one factor of agoraphobia showed an adequate fit to the data. The internal consistency values were high, distributions were positively skewed, women averaged higher than men, and correlations were low with age and the BIDR impression management factor. They were moderate with the SIAS and SSPS. We suggest the use of the SPAI-4I in Mexico and its study in other Spanish-speaking countries.

KEY WORDS: Agoraphobia; social anxiety; social desirability; social phobia.

1, 3. Facultad de Psicología, Universidad Autónoma de Nuevo León. c/Dr. Carlos Canseco 110. Col. Mitras Centro. C.P. 64460. Monterr ey, Nuevo León, México. Teléfono: (81) 8333 8233. Ext. 423 y Fax: Ext. 103. E-mail 1: jose_moral@hotmail.com,E-mail 3: garicimx@yahoo.com.mx

2. Facultad de Psicología, Universidad Pontificia de Salamanca. C/ Compañía, 5. C.P. 37002 Salamanca, España. Teléfono: 923277113 y Fax: 923277114 . Email: cjantonaca@upsa.es 
INTRODUCCIÓN

Se puede definir fobia social como el miedo persistente y acusado a situaciones sociales 0 actuaciones en público por temor a que resulten embarazosas (American Psyquiatric Association [APA], 2000). Uno de los instrumentos más empleados para evaluar ansiedad social tanto en investigación como en clínica es el Inventario de ansiedad y fobia social (SPAI) de Turner et al. (1989). El SPAl está integrado por dos factores correlacionados: fobia social con 96 ítems y agorafobia con 13 ítems. El número de factores se determinó por el criterio de Cattell (punto de inflexión de la curva de sedimentación de autovalores) y se definieron tras una rotación ortogonal. Al extraer los factores del total de 96 ítems de fobia social, Turner et al. (1989) definieron cinco: 1) ansiedad en las relaciones con el sexo opuesto y personas en general, 2) falta de asertividad ante autoridades y timidez ante extraños, 3 ) respuestas cognitivas y fisiológicas en situaciones sociales, 4) evitación/abandono y 5) ansiedad de ejecución. El número de factores se determinó por el criterio de Kaiser (autovalores mayores a 1) y se definieron nuevamente desde la matriz de componentes rotada. En este mismo estudio, los investigadores reportaron valores de estabilidad temporal $(r=.86)$ y consistencia interna $(\alpha=.96)$ altos para los 96 ítems de fobia social, al igual que para los 13 ítems de agorafobia (.85, ambos coeficientes) en muestras estadounidenses de adultos.

Los valores de consistencia interna altos y la estructura de dos factores del SPAI han sido reportados en otras investigaciones, ya sea en población estadounidense (Osman et al., 1996) u otras poblaciones, como la española (Olivares, García-López, Hidalgo, Turner y Beidel, 1999), chilena (Olivares et al., 2010) o china (Zhou, Xu, Inglés, Hidalgo y La Greca, 2008), aunque en estos estudios se requirió eliminar ítems por saturaciones bajas o contra expectativa para reproducir los dos factores. Debe mencionarse que los factores de ansiedad social varían en número e incluso de interpretación de uno a otro estudio, y que se ha hecho una propuesta de reducción de ítems a un total de 23 , desde la teoría de la respuesta al ítem, para definir con más claridad la unidimensionalidad de cada factor (Roberson-Nay, Strong, Nay, Beidel y Turner, 2007).

La ansiedad social y agorafóbica, al ser dos características desadaptativas, son poco frecuentes en población general, $y$ ambas aparecen juntas en bastantes casos clínicos (Bögels et al., 2010); de tal forma que una escala que evalúe estos rasgos de forma directa debería mostrar una distribución asimétrica positiva, con una media por encima de la mediana y moda (Sartori, 2006), y mostrar correlaciones moderadas y positivas entre los dos factores. Todos los trastornos de la dimensión afectiva, en la que se incluyen la fobia social y agorafobia, son más frecuentes en mujeres que en hombres, usualmente con prevalencia del doble de casos (Goldberg, Krueger, Andrews y Hobbs, 2009), lo que se atribuye al efecto de los descensos cíclicos de los estrógenos gonadales 0 permanentes (menopausia) sobre las indolaminas y catecolaminas (Stahl, 2001), y a diversos rasgos derivados de los procesos de socialización y construcción de género, como mayor vulnerabilidad social (dependencia económica), mayor sensibilidad interpersonal y mayor dependencia de otros para afrontar estresores (Hofmann, AnuAsnaani y Hinton, 2010). De este modo las escalas que evalúan ansiedad deberían reflejar este hecho por medio de diferencias de medias, con mayor promedio en mujeres que en hombres.

La timidez y el miedo a salir fuera de casa $\sin$ apoyo de familiares 0 alguien conocido, aunque muestran variabilidad individual, disminuyen con la edad desde la infancia a la edad adulta por la mayor 
independencia, seguridad en sí mismo y recursos con los que el adulto cuenta (Beesdo, Knappe, \& Pine, 2009); en el adulto mayor puede aumentar, sobre todo el miedo a salir solo, por la pérdida de independencia que genera el proceso fisiológico de envejecimiento (WolitzkyTaylor, Castriotta, Lenze, Stanley, \& Craske, 2010). Por lo tanto se esperaría que, en adultos, las escalas que evalúan ansiedad social tengan correlación inversa con la edad.

Diversas investigaciones muestran que la distribución del SPAl es asimétrica positiva, promedian más alto las mujeres que los hombres, correlaciona con la edad, aunque con un tamaño de efecto pequeño y sin que exista interacción entre sexo y edad (Olivares, Piqueras y Alcázar, 2006; Olivares et al., 2010). De este modo el SPAI muestra validez de constructo, conforme a las expectativas enunciadas en el párrafo previo, aunque haya algún estudio que reporte independencia con la edad (Inglés et al., 2010).

La deseabilidad social se suele contemplar como una distorsión de las respuestas en un sentido socialmente favorable que puede invalidar el autorreporte. Precisamente, el autorreporte en escalas que evalúan rasgos desadaptativos o no deseables socialmente, como son las de fobia social, puede ser afectado por este sesgo (Butcher, Dahlstrom, Graham, Tellegen y Kaemer, 1989). Paulhus (2002) distingue dos aspectos dentro de la deseabilidad social: el fingimiento 0 manejo consciente de la impresión en un sentido socialmente favorable, y el autoengaño 0 sesgo automático a atribuirse cualidades positivas que en realidad no se poseen e ignorar defectos que se tienen, engrandeciendo así la imagen del yo ante la evaluación social.

EI SPAI ha sido validado en países de habla hispana, como-España (GarcíaLópez, Olivares, Hidalgo, Beidel y Turner,
2001; Olivares et al., 1999) y Chile (Olivares et al., 2010), pero no en México. El presente estudio pretende efectuar la validación en este país a través de los siguientes objetivos: 1) contrastar una estructura de dos factores correlacionados (fobia social y agorafobia), acudiendo análisis factorial exploratorio para seleccionar los indicadores de cada factor y las posibles factores subordinados al fobia social, 2) calcular la consistencia interna del conjunto de ítems y los factores, 3) describir la distribución del puntaje diferencial y de los factores, 4) contrastar la equivalencia de la estructura factorial entre ambos sexos, pero con diferencias de medias, 5) calcular las correlaciones del SPAI con la edad, 6) comprobar la validez convergente del SPAI con dos escalas que evalúan ansiedad social y 7) estimar su relación con la deseabilidad social.

Se espera un ajuste adecuado de la estructura de dos factores correlacionados (fobia social y agorafobia) y 5 factores jerarquizados al de fobia social, probablemente tras la reducción de indicadores, siendo esta estructura válida para hombres y mujeres; consistencia interna alta; distribuciones asimétricas positivas; mayores promedios en mujeres; correlaciones bajas con la edad y moderadas 0 altas con las escalas de la ansiedad social. Al ser el SPAI una escala psicopatológica podría correlacionar con el factor de manejo de la impresión de la deseabilidad social, lo que previamente no se ha estudiado.

\section{MÉTODO}

\section{Participantes}

La población objeto de estudio fueron estudiantes. Se realizó un muestreo de cuotas equivalentes de sexos. Se trabajó con dos facultades de la Universidad Autónoma de Nuevo León: psicología (con mayoría de mujeres) y organización deportiva (con mayoría de hombres). Entre ambas suman 2,500 alumnos de 
licenciatura con equivalencia de sexos. Para estimar un tamaño de muestra representativo de la población se tomó como parámetro la media de la puntuación diferencial del SPAI calculada con sus 109 ítems. Siendo la desviación estándar esperada de 85 (Turner et al., 1989), para un error absoluto esperado de 5 y un nivel de confianza de $95 \%$, se requirió un tamaño de muestra de 695 participantes (Thrusfield, Ortega, de Blas, Noordhuizen y Frankena, 2001).

Dicha muestra quedó integrada por 314 hombres y 381 mujeres, siendo la frecuencia de sexos estadísticamente equivalente (prueba binomial: $p=.07$ ). La media de edad fue de 19.15 años $(D E=$ 2.13). De los 695 participantes, 681 (98\%) reportaron estar solteros y $14(2 \%)$ casados o en unión libre. El 52\% (361 de 695) de la muestra procedió de la facultad de psicología y $48 \%$ (334 de 695) de la facultad de organización deportiva, siendo los porcentajes estadísticamente equivalentes (prueba binomial: $p=.36$ ). El 80\% (556 de 695) dijo sólo estudiar y 20\% (139 de 695) estudiar y trabajar. El ingreso familiar mensual promedio fue 12,500 pesos $(1,000$ dólares).

\section{Instrumentos}

Inventario de ansiedad y fobia social (SPAI) de Turner et al. (1989). Lo integran 109 ítems directos que se responden en una escala tipo Likert con un rango de 7 puntos (de 1 nunca a 7 siempre). Se compone de una escala de la fobia social con 96 ítems (enumerados del 1 al 32e), y otra de agorafobia, compuesta con 13 ítems (enumerados del 33 al 45). Véase el inventario en el Anexo 1. A partir de sus 109 ítems se definen tres puntuaciones: una de fobia social, otra de agorafobia y otra denominada puntuación de diferencia; esta última se obtiene al restar la puntuación de agorafobia a la puntuación de fobia social. Esta puntuación pretende eliminar la ansiedad proveniente de la agorafobia, por lo que se considera una puntuación "pura" de fobia social.

Escala de ansiedad en la interacción social (SIAS) de Mattick y Clarke (1998). Está integrada por 20 ítems tipo Likert con un rango de respuesta de 5 puntos. De sus 20 ítems 17 están redactados en sentido de ansiedad social y 3 en sentido inverso $(5,9$ y 11). Posee consistencia interna alta ( $a=$ .88) y estructura unidimensional (Mattick y Clarke, 1998).

Escala de autoverbalizaciones al hablar en público (SSPS) de Hofmann y DiBartolo (2000). Consta de 10 ítems tipo Likert con un rango de respuesta de seis puntos que miden las autoverbalizaciones que generalmente están presentes en una situación de hablar en público. Se compone de dos factores: autoverbalizaciones positivas (SSPS-P) con 5 ítems (1, 3, 5, 6 y 9) y autoverbalizaciones negativas (SSPSN) con los 5 ítems restantes (Hofmann y DiBartolo, 2000).

Inventario balanceado de deseabilidad social al responder (BIDR) de Paulhus (1998). Consta de 40 ítems redactados como proposiciones afirmativas, la mitad en sentido de respuestas socialmente deseables y la otra mitad en sentido opuesto $(2,4,6,8,10,12$, $14,16,18,20,21,23,25,27,29,31,33,35$, 37 y 39). Se responden según una escala tipo Likert que va del 1 ("no cierto") hasta el 7 ("muy cierto"). Consta de dos factores: autoengaño (ítems del 1 al 20) y manejo de impresión (del 21 al 40). Cada factor cuenta con la mitad de sus ítems directos (atribución de cualidades) y la otra mitad en sentido inverso (negación de defectos), pudiéndose así distinguir 4 factores.

\section{Procedimiento}

Las cuatro escalas (SPAI, SIAS, SSPS y BIDR) fueron traducidas por el procedimiento de traducción reversa (García-Cadena, 2009). Primero se tradujeron al español por un experto (lingüista), luego se volvieron a traducir al 
inglés por un segundo experto (lingüista). Se evaluó la equivalencia semántica de ambas traducciones por otro tercero (psicólogo) en una escala de 5 "totalmente equivalente" a 1 "totalmente disimilares". Todos aquellos ítems con menos de 4 puntos se tradujeron de nuevo hasta lograr una puntuación de al menos 4 bajo el mismo procedimiento. A continuación se evaluó la comprensibilidad de las escalas en una muestra de 30 participantes con estudios de primaria. La escala de evaluación fue de tres puntos: $3=$ "comprensible", 2 = "genera dudas", 1 = "no lo comprendo". Se exigió para cada ítem que al menos 25 de los 30 participantes (80\%) señalen comprenderlo (puntuación 3). En caso de marcar 1 ó 2 se pidió indicar por qué. A partir de los señalamientos se hicieron modificaciones.

El tiempo en cumplimentarlas fue de unos 30 minutos. Para evitar el efecto de la fatiga y orden fijo de secuencia en los resultados de cada escala se empleó el método de equiponderación total.

El trabajo de campo fue realizado por alumnos de un curso de investigación en psicología, tras recibir una capacitación, de junio a diciembre de 2010. La aplicación fue individual para garantizar la independencia de los participantes, los cuales fueron seleccionados de forma aleatoria en los jardines y áreas públicas de las dos facultades.

Se respetaron las recomendaciones éticas de la American Psychological Association (2002); así la participación en el estudio fue voluntaria y anónima; se requirió proporcionar el consentimiento expreso en la primera página del cuestionario, garantizándose la confidencialidad de la información; y en caso de que algún participante solicitase ayuda psicológica, éste era derivado a la unidad clínica de la facultad. El proyecto de investigación fue aprobado por el Comité de Ética de la Facultad de Psicología de la Universidad
Autónoma de Nuevo León, la cual financió este estudio.

Análisis de datos

La consistencia interna se estimó por el coeficiente alfa de Cronbach (a). El ajuste de la distribución a una curva normal se contrastó por la prueba de KolmogorovSmirnov $\left(Z_{K-S}\right)$. Los promedios entre hombres y mujeres se compararon por la prueba $t$ de Student para dos muestras independientes. Las correlaciones entre las escalas y con la edad se calcularon por el coeficiente producto-momento de Pearson. La estructura dimensional esperada se contrastó por análisis factorial confirmatorio por Mínimos Cuadrados Generalizados. Se optó por este método a ser más robusto a la violación de supuesto de normalidad que Máxima Verosimilitud y permitir la estimación de la significación de los parámetros frente a Mínimos Cuadrados Ordinales que no. La curtosis multivariada de Mardia fue mayor a 70 (Rodríguez \& Ruíz, 2008). Se contemplaron cuatro índices de ajuste: cociente entre el estadístico chicuadrado y sus grados de libertad ( $2 / g h)$, residuo cuadrático medio de aproximación (RMSEA) de Steiger-Lind, índice de bondad de ajuste (GFI) de Jöreskog y Sörbom y su modalidad corregida (AGFI). Se estipularon como valores de buen ajuste para los índices: $X 2 / g l \leq 2, R M S E A \leq .05, G F I \geq .95$ y $A G F I \geq .90 ;$ y como valores adecuados: $\mathrm{X} 2 / g \mathrm{I} \leq 3, \mathrm{RMSEA} \leq .07, \mathrm{GFI} \geq .85$ y $\mathrm{AGFI}$ $\geq .80$ (Moral, 2006). Los contrastes se hicieron tanto por la modalidad unigrupo como multigrupo (ambos sexos). Para mejorar el ajuste se exploró modelos factoriales alternativos por Componentes Principales con rotación Oblimín, buscando definir los factores subyacentes a la dimensión de fobia social y los mejores indicadores de fobia social y agorafobia (con saturaciones mayores a .50). Para delimitar el número de factores se consideraron los criterios de la expectativa, Kaiser, Cattell y Horn (punto de intersección entre la curva de sedimentación de los autovalores 
observados y la curva correspondiente al percentil 95 de los 100 ensayos con 109 variables aleatorias de distribución normal con 695 casos cada una). Para comparar la bondad de ajuste de los modelos se empleó la prueba de la diferencia de los estadísticos chi-cuadrado (Yuan y Bentler, 2004). Se introducen correlaciones entre residuos tras revisar los índices de mejora del ajuste (> 10) que suelen revelar factores subyacentes o errores de muestreo (Byrne, 2010). Los cálculos estadísticos se realizaron con el SPSS16, AMOS16 para factorial confirmatorio y VISTAS7 para el criterio de Horn (Young, 2010).

\section{RESULTADOS}

Estructura factorial por componentes principales y consistencia interna

Si se fuerza la solución de los 109 ítems a dos componentes, con base en la expectativa (Turner et al., 1989) y el criterio de Horn (1.94 es el punto de intersección de la curva de autovalores observados y la curva correspondiente al percentil 95 de los 100 ensayos con 109 variables aleatorias de distribución normal con 695 casos cada una), se obtiene una solución que se asemeja a la esperada. Para ajustarnos más a las expectativas se eliminaron 16 ítems ambiguos, esto es, con saturaciones mayores a .30 en ambos componentes y una diferencia entre ambas saturaciones menor a .10 (ítems 9d, 10d, 11c, 12d, 20a, 20b, 20c, 24a, 26a, 26b, 26d, 26e, 30a, 30c, $32 \mathrm{~b}$ y 34), 2 ítems con saturaciones bajas en ambos componentes, es decir, menores a .30 (ítems 11d y 43) y 25 ítems que contravinieron las expectativas sin ambigüedad (ítems 7, 8, 20d, 21a, 21b, 21c, 21d, 24b, 24c, 24d, 25a, 25b, 25c, 25d, 26c, $28,30 \mathrm{~b}, 30 \mathrm{~d}, 31 \mathrm{a}, 31 \mathrm{~b}, 31 \mathrm{c}, 32 \mathrm{a}, 32 \mathrm{c}, 32 \mathrm{~d}$ y $32 \mathrm{e})$.

Al extraer dos componentes de la matriz de correlaciones de los 66 ítems seleccionados, se explica el $49.02 \%$ de la varianza total. El número de dos componentes se justifica por el criterio de Cattell, al ubicarse el punto de inflexión de la curva de sedimentación en el tercer autovalor, y de nuevo por el criterio de Horn (1.96). El primer componente de 55 ítems con saturaciones mayores a $.50 \mathrm{y}$ consistencia interna alta $(\alpha=.98)$ es de fobia social. El segundo de 11 ítems con saturaciones mayores a $.50(33,35,36,37$, $38,39,40,41,42,44$ y 45$)$ y consistencia interna alta $(\alpha=.89)$ es de agorafobia. La correlación entre ambos componentes es moderada $(r=.46, p<.01)$.

Al extraer cinco componentes entre los 96 ítems de fobia social, conforme a la expectativa, se explica el $55.71 \%$ de la varianza total. Tras una rotación Oblimín, el primer componente queda configurado por 22 ítems con saturaciones mayores 0 iguales a .31 (9a, 10a, 11a, 12a, 12b, 13a, 13b, 14a, 14b, 15a, 15b, 15d, 16a, 16b, 17a, $17 b, 18 a, 18 b, 19 a, 19 b, 22 a$ y 22b), tiene consistencia interna alta $(a=.96)$ y se puede interpretar como timidez ante figuras de autoridad y extraños; el segundo componente está conformado por 10 ítems con saturaciones mayores 0 iguales a .40 $(7,8,24 a, 24 b, 24 c, 24 d, 25 a, 25 b, 25 c$ y $25 \mathrm{~d}$ ), tiene consistencia interna alta ( $a=$ .93) y se puede interpretar como evitación/abandono de situaciones sociales generadoras de ansiedad; el tercero está definido por 24 ítems con saturaciones mayores o iguales a .37 (23a, 23b, 23c, 23d, 26a, 26b, 26c, 26d, 26e, 27, 28, 29, 30a, 30b, 30c, 30d, 31a, 31b, 31c, 32a, 32b, 32c, $32 d$ y $32 e$ ), tiene consistencia interna alta (a $=.95$ ) y se puede interpretar como miedos y reacciones fisiológicas ante situaciones sociales; el cuarto está formado por 29 ítems con cargas iguales o mayores a .30 $(1,2,3,4,5,6,9 b, 9 c, 9 d, 10 b, 10 c, 10 d$, $11 \mathrm{~b}, 11 \mathrm{c}, 11 \mathrm{~d}, 12 \mathrm{c}, 12 \mathrm{~d}, 13 \mathrm{c}, 13 \mathrm{~d}, 14 \mathrm{c}, 14 \mathrm{~d}$, 15c, 16c, 16d, 18c, 18d, 19c, 22c y 22d), tiene consistencia interna alta $(\alpha=.96)$ y se puede interpretar como ansiedad en relaciones interpersonales con el sexo opuesto y personas general; finalmente, el quinto está conformado por 11 ítems (17c, 
17d, 19d, 20a, 20b, 20c, 20d, 21a, 21b, $21 \mathrm{c}$ y $21 \mathrm{~d}$ ), tiene consistencia interna alta (a $=.92$ ) y se puede interpretar como ansiedad de ejecución ante situaciones sociales que impliquen escribir, comer o tomar. Los 5 componentes tienen correlaciones moderadas entre sí. Al calcular las puntuaciones factoriales por el método de regresión y extraer los componentes de su matriz de correlaciones, se define sólo un componente por el criterio de autovalores mayores a 1, explicándose el $49.33 \%$ de la varianza total.

Si nos quedamos con 46 ítems, aquéllos con cargas mayores a .50 , se puede justificar el número de componentes por el segundo punto de inflexión de la curva de sedimentación, pero no así por el criterio de Kaiser (8) ni el de Horn (2.03). Con cinco factores se explica el $64.06 \%$ de la varianza total. Quedarían con 10 ítems los componentes de ansiedad ante relaciones interpersonales con el sexo opuesto $y$ personas en general $(9 c, 9 d, 10 c, 10 d, 11 c$, 12c, 12d, 13c, 13d y 16c; $a=.93)$, timidez ante figuras de autoridad y extraños (13a, $13 \mathrm{~b}, 14 \mathrm{a}, 14 \mathrm{~b}, 15 \mathrm{a}, 15 \mathrm{~b}, 18 \mathrm{a}, 18 \mathrm{~b}, 19 \mathrm{a}$ y $19 \mathrm{~b} ; \mathrm{a}=.93)$ y miedos y reacciones fisiológicas ante situaciones sociales $(26 \mathrm{~b}$, 26c, 26d, 26e, 30b, 30c, 31a, 31c, 32b y 32e; $a=.91$ ); y con 8 ítems quedarían los componentes de ansiedad de ejecución al escribir, comer 0 beber en situaciones sociales (20a, 20b, 20c, 20d, 21a, 21b, 21c y $21 d ; a=.92)$ y evitación/abandono de situaciones sociales generadoras de ansiedad (24a, 24b, 24c, 24d, 25a, 25b, 25c y $25 \mathrm{~d}$; $a=.93$ ). Al calcular las puntuaciones factoriales por el método de regresión y extraer los componentes de su matriz de correlaciones, se define uno por el criterio autovalores mayores a 1, explicándose el $53.29 \%$ de la varianza total.

\section{Análisis factorial confirmatorio}

Se contrasta el modelo de dos factores correlacionados. El primero de fobia social con 55 indicadores y el segundo de agorafobia con 11 indicadores. Todos los parámetros son significativos. La correlación entre los dos factores es moderada alta $(r=$ .66). Los índices de bondad de ajuste son malos $(X 2[2078, N=695]=6682.26, X 2 / g l=$ $3.22, \mathrm{GFI}=.71, \mathrm{AGFI}=.69$ y $R M S E A=.06$ ) (véase Tabla 1) y próximos al modelo independiente $(X 2[2145, \quad N=695]=$ $7200.14, X 2 / g l=3.36, G F I=.69, A G F I=.68$ y RMSEA = .06).

TABLA 1.

Ajuste de ocho modelos contrastados por mínimos cuadrados generalizados.

\begin{tabular}{lccccccccc}
\hline & \multicolumn{10}{c}{ AG-1F } & \multicolumn{9}{c}{ FS-5J } & \multicolumn{3}{c}{ SPAl-2F } & \multicolumn{2}{c}{ SPAl-2F-5J } \\
\cline { 2 - 10 } & 11 & 11 & 42 & 30 & 30 & 66 & 41 & 41 & 41 \\
\hline Ítems & 66 & 66 & 903 & 465 & 465 & 2211 & 861 & 861 & 861 \\
Momentos & 22 & 26 & 89 & 65 & 68 & 133 & 83 & 88 & 93 \\
Parámetros & 0 & 4 & 0 & 0 & 3 & 0 & 0 & 0 & 5 \\
$r_{\text {residuos }}$ & 225.90 & 125.15 & 3397.08 & 1801.89 & 1571.97 & 6728.86 & 3323.83 & 2488.95 & 2176.41 \\
X2 & 44 & 40 & 814 & 400 & 397 & 2078 & 778 & 773 & 768 \\
gl & $<.01$ & $<.01$ & $<.01$ & $<.01$ & $<.01$ & $<.01$ & $<.01$ & $<.01$ & $<.01$ \\
$p$ & 5.13 & 3.13 & 4.17 & 4.50 & 3.96 & 3.24 & 4.27 & 3.22 & 2.83 \\
X2/gl & .94 & .97 & .77 & .83 & .85 & .71 & .77 & .83 & .85 \\
GFI & .91 & .95 & .74 & .80 & .82 & .69 & .74 & .81 & .83 \\
AGFI & .08 & .05 & .07 & .07 & .07 & .06 & .07 & .06 & .05 \\
RMSEA & .07 &
\end{tabular}

Modelos: AG-1F: Un factor de agorafobia con 11 indicadores, FS-5J: 5 factores subordinadas a uno general de fobia social, primero con 42 ítems, segundo con 30 ítems y tercero con 30 ítems introduciendo 3 correlaciones entre residuos; SPAI-2F: Modelo de 2 factores correlacionados, primero con 66 ítems: agorafobia con 11 indicadores y fobia social con 45 indicadores, y segundo con 41 ítems: agorafobia con 11 indicadores y fobia social con 39 indicadores; SPAI-2F-5J: Modelo de dos factores correlacionados, agorafobia con 11 indicadores y fobia social con 5 factores subordinados, primero sin correlaciones entre residuos y segundo con 5 correlaciones entre residuos. 
El ajuste del modelo unidimensional para los 11 ítems de agorafobia presenta unos índices de ajuste mucho mejores (véase Tabla 1). Al introducir 4 correlaciones entre residuos, el ajuste es bueno para los índices de Joreskog-Sorbom (GFI $=.96$ y $A G F I=$ .93), adecuado por el error de aproximación cuadrático medio (RMSEA $=.06)$, aunque el cociente entre el estadístico chi-cuadrado y sus grados de libertad resulta ligeramente alto $(X 2[40, N=695]=148.40, X 2 / g l=3.71)$, estando estos 4 índices claramente alejados del modelo independiente $(X 2[55, N=695]=$ 3283.59, $\mathrm{X} 2 / \mathrm{gl}=59.70, \mathrm{GFI}=.33, \mathrm{AGFI}=$ .19 y $R M S E A=.29)$.

Se contrasta el modelo cinco factores (ansiedad ante relaciones interpersonales con el sexo opuesto y personas en general con 10 indicadores; timidez ante figuras de autoridad y extraños con 10 indicadores; miedos y reacciones fisiológicas ante situaciones sociales con 10 indicadores; ansiedad de ejecución al escribir, comer 0 beber en situaciones sociales con 8 indicadores; y evitación/abandono de situaciones sociales generadoras de ansiedad con 8 indicadores) jerarquizados a otro de orden superior (fobia social). Este modelo fue obtenido a partir del análisis factorial exploratorio y se asemeja al de Turner et al. (1989).

Al ser calculado, la minimización no es exitosa por problemas en el factor de ansiedad de ejecución, el cual requiere ser reducido a las cuatro variantes del ítem 20 (comer 0 beber en público), eliminándose las 4 variantes del ítem 21 (escribir en público). Así, este factor pasa a ser denominado ansiedad al comer o beber en público. Con los 42 ítems restantes el ajuste es malo $(X 2[814, N=695]=3381.61, X 2 / g l$ $=4.15, \mathrm{GFI}=.77, \mathrm{AGFI}=.74$ y $R M S E A=$ $.07)$.

Con el objetivo de mejorar el ajuste se reduce el número de indicadores a 30 . El factor de miedos y reacciones fisiológicas queda en 6 ítems (26b, 26c, 26d, 26e, 30b y
$30 \mathrm{c} ; \mathrm{a}=.89$ ), relaciones interpersonales en 8 ítems (9c, 9d, 10c, 12c, 12d, 13c, 13d y $16 \mathrm{c} ; \mathrm{a}=.93)$, timidez con las autoridades $\mathrm{y}$ extraños en 4 ítems (18a, 18b, 19a, 19b; $a=$ $.91)$, comer y beber en público en 4 (21a, 21b, 22c y 22d; a $=.93$ ) y evitación/abandono conserva sus 8 ítems (24a, 24b, 24c, 24d, 25a, 25b, 25c y 25d; a $=.93)$. El ajuste mejora significativamente (dx2[414, $N=695]=1595.19, p<.01)$, y al introducir cuatro correlaciones entre residuos se logra un ajuste adecuado $(X 2[455, N=695]=1339.25, X 2 / g l=2.94$, $\mathrm{GFI}=.85, A G F I=.82$ y $R M S E A=.06$ ) (véase Tabla 1). Debe señalarse que con estos 30 ítems, al extraer los factores por componentes principales, se definen 5 por el criterio de Kaiser, se explica el $71.97 \%$ de la varianza total y la consistencia interna es .96 .

Al contrastar un modelo de un factor general con 5 jerarquizados para 30 ítems de fobia social y de un factor general para 11 ítems de agorafobia, estando correlacionados el factor general de fobia social y agorafobia, se obtiene un ajuste significativamente mejor que al considerar sólo dos factores correlacionados (dx2[5, N $=695]=834.88, p<.01)$. Al introducir 5 correlaciones entre residuos (4 en el factor general de fobia social y 1 en agorafobia) los valores de los 4 índices son adecuados: $\mathrm{X} 2[768, N=695]=2176.79, \mathrm{X} 2 \mathrm{gl}=2.83$, GFI $=.85, A G F I=.83$ y RMSEA $=.05$ (véase Tabla 1). Todos los parámetros son significativos y la correlación entre el factor general de fobia social y agorafobia es alta $(r=.73)$, habiendo un $53 \%$ de varianza compartida (véase Figura 1).

Al realizar el contraste (del modelo sin restricciones) entre ambos sexos el ajuste es de adecuado (X2[1538, $N=695]=$ 3298.62, $\mathrm{X} 2 / \mathrm{gl}=2.14, \mathrm{GFI}=.87, \mathrm{AGFI}=$ $.84)$ a bueno (RMSEA $=.04)$, siendo todos los parámetros significativos en ambas muestras. 
FIGURA 1.

Modelo estandarizado de cinco factores jerarquizados a uno general de fobia social (con un total de 30 ítems) correlacionado a un factor general de agorafobia (con 11 indicadores) estimado por Mínimos Cuadrados Generalizados.

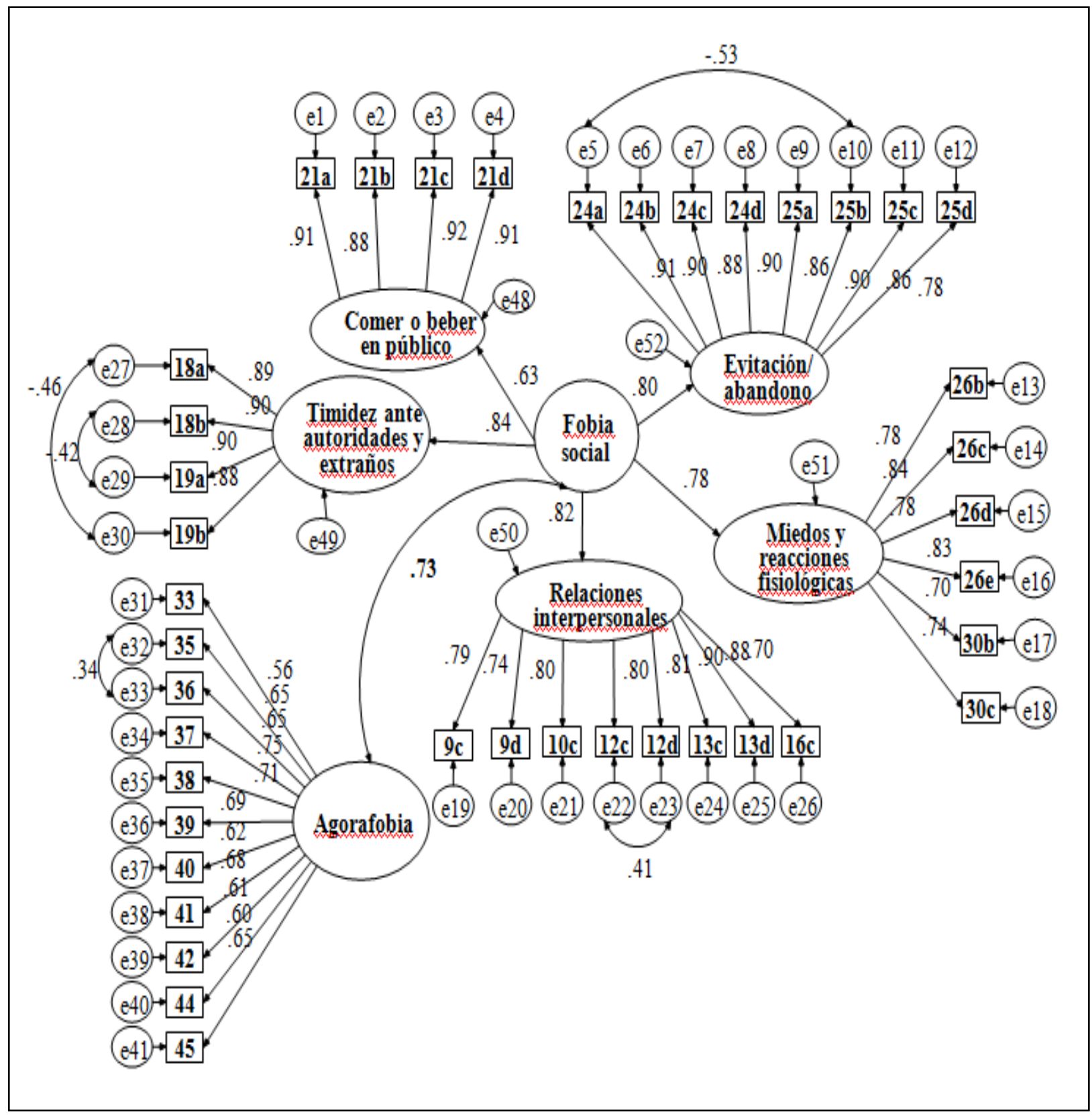

Distribución, diferencias de medias entre sexos y correlación con la edad

Con base en el resultado del análisis factorial confirmatorio, se propone reducir el SPAI a 41 ítems. Las distribuciones de la puntación diferencial del inventario, de las dos escalas y de los cinco factores no se ajustan a una curva normal. Son asimétricas positivas $(S>.18)$ y apuntadas $(C>.36)$, concentrándose en los valores bajos (véase Tabla 2). 
TABLA 2.

Estadísticos descriptivos y ajuste a la normalidad de las distribuciones del SPAI-41.

\begin{tabular}{|c|c|c|c|c|c|c|c|c|c|}
\hline \multirow{2}{*}{ Estadísticos } & & \multicolumn{8}{|c|}{ SPAl-41 } \\
\hline & & PD & FS & ARI & TAE & CBP & EVA & MRF & AG \\
\hline \multicolumn{2}{|l|}{ Mínimo } & 3 & 30 & 8 & 4 & 4 & 8 & 6 & 11 \\
\hline \multicolumn{2}{|l|}{ Máximo } & 145 & 210 & 56 & 28 & 28 & 56 & 42 & 76 \\
\hline \multicolumn{2}{|l|}{ Media } & 42.39 & 60.28 & 16.30 & 10.11 & 6.75 & 13.92 & 13.21 & 17.90 \\
\hline \multicolumn{2}{|c|}{ Desviación estándar } & 22.86 & 28.02 & 8.64 & 5.69 & 4.72 & 8.46 & 7.34 & 9.45 \\
\hline \multicolumn{2}{|c|}{ Sesgo } & 1.28 & 1.48 & 1.38 & 1.15 & 2.19 & 1.93 & 1.47 & 2.39 \\
\hline \multirow[t]{2}{*}{ Curtosis } & & 1.61 & 2.52 & 2.05 & 0.89 & 4.72 & 4 & 2.07 & 7.20 \\
\hline & 25 & 25 & 39 & 9 & 6 & 4 & 8 & 8 & 11 \\
\hline \multirow[t]{2}{*}{ Percentiles } & 50 & 37 & 53 & 14 & 8 & 4 & 10 & 11 & 14 \\
\hline & 75 & 53 & 71 & 21 & 13 & 8 & 17 & 17 & 20 \\
\hline Prueba de & $D$ & 0.12 & 0.14 & 0.17 & 0.15 & 0.29 & 0.24 & 0.16 & 0.23 \\
\hline Kolmogorov- & $Z$ & 3.13 & 3.69 & 4.44 & 3.95 & 7.60 & 6.38 & 4.32 & 6.14 \\
\hline Smirnov & $p$ & $<.01$ & $<.01$ & $<.01$ & $<.01$ & $<.01$ & $<.01$ & $<.01$ & $<.01$ \\
\hline
\end{tabular}

$\mathrm{N}=695$. El error estándar del sesgo es de .09 y de la curtosis de .18. SPAl-41: PD = Puntación diferencial (FS - AG), FS = Escala de fobia social reducida a 30 ítems, $A R I=$ Factor de ansiedad en relaciones interpersonales con personas del sexo opuesto y personas en general, TAE = Factor de timidez ante autoridades y extraños, CBP = Factor de comer y beber en público, $E V A=$ Factor de evitación/abandono de situaciones sociales generadoras de ansiedad, MRF $=$ Factor de miedos y reacciones fisiológicas y $A G=$ Escala de agorafobia reducida a 11 ítems

Las medias de las mujeres fueron significativamente mayores en las escalas de agorafobia y fobia social, así como en los factores de ansiedad en relaciones interpersonales con el sexo opuesto y personas en general, timidez ante autoridades y extraños y miedos $y$ reacciones fisiológicas (véase Tabla 3).

TABLA 3.

Contraste de medias en el SPAI-41 entre mujeres y hombres.

\begin{tabular}{cccccccccc}
\hline \multirow{2}{*}{ SPAl-41 } & \multicolumn{3}{c}{ Mujeres $(n=372)$} & \multicolumn{3}{c}{ Hombres $(n=323)$} & \multicolumn{3}{c}{ Contraste de medias } \\
\cline { 2 - 9 } & $M$ & $D E$ & $E E M$ & $M$ & $D E$ & $E E M$ & $t$ & $g l$ & Sig. \\
\hline PD & 43.87 & 24.05 & 1.25 & 40.69 & 21.33 & 1.19 & -1.85 & 692.68 & $n s$ \\
FS & 62.83 & 29.09 & 1.51 & 57.35 & 26.47 & 1.47 & -2.60 & 691.46 & $<.05$ \\
ARI & 17.30 & 9.32 & 0.48 & 15.15 & 7.65 & 0.43 & -3.33 & 690.94 & $<.01$ \\
TAE & 10.92 & 5.77 & 0.30 & 9.19 & 5.45 & 0.30 & -4.07 & 688.10 & $<.01$ \\
CBP & 6.92 & 4.93 & 0.26 & 6.56 & 4.46 & 0.25 & -0.99 & 693 & $n s$ \\
EVA & 13.97 & 8.61 & 0.45 & 13.85 & 8.29 & 0.46 & -0.19 & 693 & $n s$ \\
MRF & 13.73 & 7.75 & 0.40 & 12.61 & 6.80 & 0.38 & -2.02 & 692.90 & $<.05$ \\
AG & 18.97 & 9.46 & 0.49 & 16.67 & 9.31 & 0.52 & -3.22 & 693 & $<.01$ \\
\hline
\end{tabular}

Cuando los grados de libertad ( $\mathrm{gl}$ ) son iguales a 693 se asume igualdad de medias y cuando son menores a 693 no se asume igualdad de medias por la prueba de Levene $(p<.05)$. SPAI-41: PD = Puntación diferencial (FS - AG), FS = Escala de fobia social reducida a 30 ítems, ARI = Factor de ansiedad en relaciones interpersonales con personas del sexo opuesto y personas en general, $\mathrm{TAE}=$ Factor de timidez ante autoridades y extraños, $\mathrm{CBP}=$ Factor de comer y beber en público, EVA $=$ Factor de evitación/abandono de situaciones sociales generadoras de ansiedad, MRF = Factor de miedos y reacciones fisiológicas y $A G=$ Escala de agorafobia reducida a 11 ítems.

Las correlaciones de la puntuación diferencial, las escalas de fobia social y agorafobia y los factores de miedos y reacciones fisiológicas, comer y beber en público, así como evitación/abandono son significativas, bajas y negativas con la edad (véase Tabla 4). 
Validez convergente y relación con deseabilidad social

En la presente muestra la consistencia interna de los 10 ítems del SSPS es alta (a $=.70)$ y la distribución del sumatorio es asimétrica positiva. Al eliminar los 3 ítems inversos del SIAS mejora la consistencia interna de .82 a .90 y el ajuste al modelo unidimensional esperado; por lo que se maneja un puntaje total de 17 ítems que tiene una distribución asimétrica positiva.

La media de las correlaciones del SPAI41 con el SIAS17 es de .56, variando de .43 a .69; y con el SSPS es de .38, variando de .29 a .47. Dentro de los factores de fobia social, destaca por sus valores de correlación, el de de ansiedad en relaciones interpersonales con personas del sexo opuesto y personas en general; por el contrario, el menos correlacionado es el factor de comer y beber en público. El patrón de correlación es el mismo en los cinco factores (véase Tabla 4).
En la presente muestra la consistencia interna de los 4 factores del BIDR varió de .61 a .76. Por el método de Mínimos Cuadrados Generalizados los índices de ajuste mejoraron al considerar sólo los 10 ítems directos de autoengaño y los 10 directos de manejo de la impresión para definir un modelo de dos factores correlacionados, siendo los valores de consistencia interna de estos dos factores mayores a .70. Así, se empleó esta versión simplificada (BIDR-20), como recomiendan Moral, García y Antona (2012).

Las correlaciones con el puntaje total del BIDR (20 ítems directos) son bajas y negativas, variando de -.02 a -.16 con una media de -.10 , siendo más altas con su factor de manejo de la impresión, variando de -.06 a -.20 con una media de -.15, cuando con el factor de autoengaño no son significativas (véase Tabla 4).

TABLA 4

Correlaciones del SPAI-41 con SIAS-17, SSPS, BIDR-20 y la edad.

\begin{tabular}{|c|c|c|c|c|c|c|}
\hline \multirow[b]{2}{*}{ SPAI-41 } & \multicolumn{2}{|c|}{ Ansiedad social } & \multicolumn{3}{|c|}{ Deseabilidad social } & \multirow[b]{2}{*}{$\begin{array}{c}\text { Edad } \\
(n=695)\end{array}$} \\
\hline & $\begin{array}{c}\text { SIAS17 } \\
(n=671)\end{array}$ & $\begin{array}{c}\text { SSPS } \\
(n=681)\end{array}$ & $\begin{array}{l}\text { BIDR-20 } \\
(n=683)\end{array}$ & $\begin{array}{c}\mathrm{AE} \\
(n=689)\end{array}$ & $\begin{array}{c}\mathrm{Ml} \\
(n=686)\end{array}$ & \\
\hline PD & $.64^{* *}$ & $.39^{* *}$ & $-.10^{* *}$ & $-.15^{* *}$ & -.01 & $-.10^{*}$ \\
\hline FS & $.69^{* *}$ & $.46^{* *}$ & $-.13^{* *}$ & $-.18^{* *}$ & -.02 & $-.11^{* *}$ \\
\hline ARI & $.61^{* *}$ & $.39^{* *}$ & $-.12^{* *}$ & $-.18^{* *}$ & -.02 & -.05 \\
\hline TAE & $.53^{* *}$ & $.29^{* *}$ & -.02 & -.06 & .03 & -.06 \\
\hline CBP & $.43^{* *}$ & $.29^{* *}$ & $-.09^{*}$ & $-.14^{* *}$ & -.02 & $-.09^{*}$ \\
\hline EVA & $.51^{* *}$ & $.38^{* *}$ & $-.09^{*}$ & $-.14^{* *}$ & -.02 & $-.09^{*}$ \\
\hline MRF & $.62^{* *}$ & $.44^{* *}$ & $-.16^{* *}$ & $-.20^{* *}$ & -.06 & $-.13^{* *}$ \\
\hline AG & $.49^{* *}$ & $.41^{* *}$ & $-.13^{* *}$ & $-.17^{* *}$ & -.04 & $-.08^{*}$ \\
\hline \multicolumn{7}{|c|}{$\begin{array}{l}{ }^{* *} \mathrm{p}<.01,{ }^{*} \mathrm{p}<.05 . \mathrm{SPAI}-41: \mathrm{PD}=\text { Puntación diferencial (FS - AG), FS = Escala de fobia social reducida a } 30 \text { ítems, ARI = Factor } \\
\text { de ansiedad en relaciones interpersonales con personas del sexo opuesto y personas en general, TAE = Factor de timidez ante } \\
\text { autoridades y extraños, CBP = Factor de comer y beber en público, EVA = Factor de evitación/abandono de situaciones sociales } \\
\text { generadoras de ansiedad, MRF = Factor de miedos y reacciones fisiológicas y AG = Escala de agorafobia reducida a } 11 \text { ítems. } \\
\text { SIAS17 = Escala de Ansiedad en la Interacción Social reducida a } 17 \text { ítems (sin los } 3 \text { ítems inversos), SSPS = Escala de } \\
\text { Autoverbalizaciones al Hablar en Público con sus } 10 \text { ítems originales, BIDR-20 = Inventario Balanceado de Deseabilidad Social al al } \\
\text { Responder reducido a sus } 20 \text { ítems directos con sus factores de autoengaño (AE) y manejo de la impresión (MI). }\end{array}$} \\
\hline
\end{tabular}




\section{DISCUSIÓN}

El modelo factorial propuesto con un ajuste adecuado en esta muestra de estudiantes mexicanos corrobora la estructura de dos factores correlacionados de la propuesta original de Turner et al. (1989), validada en una muestra estadounidense por Osman et al. (1996), en España por Olivares et al. (1999), en Chile por Olivares et al. (2010) y en China por Zhou et al. (2008); asimismo, retoma la propuesta inicial de Turner et al. (1989) de una estructura de cinco factores para los ítems de fobia social. Aunque posteriormente se han considerado cuatro factores para los ítems de fobia social (García-López et al., 2001), dicha estructura resulta más inestable en esta muestra, al igual que cualquier modelo unidimensal reducido en indicadores, como sugieren Roberson-Nay et al. (2007) con los datos presentes (omitidos por cuestión de espacio).

Como en el presente, en todos los estudios posteriores al de Turner et al. (1989) se ha querido reducir el número de ítems para ajustarse a las expectativas con este inventario, lo que indica que tiene un número excesivo de ítems.

El modelo de este estudio propone un factor general de fobia social que determina cinco factores subordinados y que correlaciona con un factor general de agorafobia con 11 indicadores. Este modelo es novedoso en la investigación de este inventario, ya que en estudios anteriores no se había realizado tal integración. Con 41 ítems (30 de fobia general y 11 de agorafobia) dicho modelo se puede reproducir tanto por análisis factorial exploratorio como confirmatorio, siendo válido para ambos sexos. Además conserva validez de contenido al evaluar tanto respuestas en el plano cognitivo, emocional, fisiológico y comportamental como escenarios con extraños, figuras de autoridad, gente desconocida y sexo opuesto.
La definición de un modelo general de ansiedad, siguiendo los hallazgos de la meta-dimensión de trastornos afectivos (Goldberg et al., 2009), generaría un modelo con dificultades de especificación que requeriría fijar en 1 los parámetros de los dos indicadores de ese factor general (fobia social y agorafobia). Una vez fijados la solución obtenida es admisible y daría un $98 \%$ de varianza explica de agorafobia sin varianza residual significativa; de ahí que se opte por el modelo de dos factores correlacionados.

El factor foco de atención de GarcíaLópez et al. (2001) se convierte en los factores timidez ante autoridades y extraños y ansiedad de ejecución; éste último, finalmente, queda simplificado como ansiedad ante situaciones que impliquen comer 0 beber en público. Los cinco factores de fobia social tienen una alta consistencia interna, a pesar de contar con un número relativamente pequeño de indicadores (de 4 a 8). Esta alta consistencia interna es una constante en otros muchos estudios (García-López et al., 2001; Olivares et al., 2010; Osman et al., 1996; Turner et al., 1989; Zhou et al., 2008), que en gran parte sustenta el factor general de ansiedad.

Conforme a las expectativas, las distribuciones no se ajustan al modelo de una curva normal, aún cuando la escala de fobia social cuenta con un número grande de ítems en su integración, lo que remarca su asimetría (Olivares et al., 2006). Por lo tanto, el inventario debería ser estandarizado por los percentiles. Las puntuaciones se concentran en los valores bajos, lo que es característico de escalas que evalúan aspectos psicopatológicos. También este fenómeno se da en rasgos 0 conductas condenadas socialmente y, por lo tanto, contaminadas por el sesgo de la deseabilidad social (Paulhus, 2002). En la presente muestra el SPAI sí correlaciona con la deseabilidad social, pero la varianza compartida es menos del $2 \%$, lo que permite 
atribuir la asimetría a la evaluación de un rasgo psicopatológico.

En estudios epidemiológicos de población general las mujeres sufren más de fobia social y agorafobia (APA, 2000; Medina-Mora et al., 2003). En las investigaciones con el SPAI las mujeres presentan niveles significativamente más altos en la escala de ansiedad social que los varones, tanto en muestras clínicas (Inglés et al., 2008; Piqueras, Olivares y LópezPina, 2008) como no clínicas (Inglés et al., 2010; Olivares et al., 2006; Zhou et al., 2008). En este estudio se confirma un promedio mayor en mujeres tanto en ansiedad social como en agorafobia. También se confirma una correlación débil con la edad, como en los estudios de Olivares et al. (2006), Olivares et al. (2010) frente al de Inglés et al. (2010), en el que se halló independencia. A mayor edad, estos estudiantes reportan menos ansiedad social y agorafobia.

Como antes se señaló, el SPAI correlaciona con deseabilidad social con un valor bajo y negativo, siendo el manejo de la impresión el que determina esta relación, ya que el factor de autoengaño resulta independiente. La correlación es negativa, por lo tanto, los más sinceros son los que puntúan más en el SPAI y los menos sinceros tienden a puntuar menos. Esto evidencia que las respuestas a unas preguntas con bastante validez aparente son susceptibles de ligera manipulación con el objetivo de mejorar la impresión social; no obstante, al ser el efecto muy pequeño, puede ser ignorado.

Las correlaciones del SPAI-41 son moderadas con el SIAS17 y más altas que con el SSPS que en general también son moderadas. Precisamente, en anteriores estudios se reportaron correlaciones moderadas, esto es, menores a $.70 \mathrm{y}$ mayores a .30 (Mattick y Clarke, 1998; Olivares, García-López, Hidalgo y Caballo, 2004). La menor correlación con el SSPS se explica porque esta escala abarca un contenido más específico, como es la ansiedad al hablar en público, cuando el contenido de ansiedad social es más amplio en el SIAS. De forma congruente a los contenidos, la escala de fobia social del SPAI-41 presenta correlaciones más altas con el SIAS17 y SSPS que la escala de agorafobia. Asimismo, el patrón de correlación es el mismo en los cinco factores que integran de forma directa el factor general de fobia social, lo que arroja evidencias de validez convergente adicionales. Se podrían justificar estas correlaciones moderadas con el SIAS17 y SSPS en lugar de altas por el matiz diferencial entre fobia (más patológico) y ansiedad social (más cotidiano).

Debe señalarse que, en la presente muestra, los promedios de mujeres y hombres son equivalentes en el SIAS y SSPS y el autoengaño tiene más peso que el manejo de la impresión en estas dos escalas; por el contrario, las mujeres promedian más alto en el SPAI (la prevalencia de todos los trastornos de ansiedad es más frecuente en mujeres; APA, 2000) y el manejo de la impresión tiene más peso. Lo que remarca que sí evalúan aspectos algo distintos de la ansiedad social, teniendo lo patológico (socialmente no deseable) un mayor peso en el SPAI.

Como limitaciones del estudio debe señalarse la muestra no probabilística, así las conclusiones deben manejarse como hipótesis para estudios posteriores, sobre todo más aplicables a estudiantes universitarios.

En conclusión, en esta muestra de estudiantes universitarios, un modelo estructural de un factor general de fobia social (con 5 factores subordinados) correlacionado con otro factor general de agorafobia muestra un ajuste adecuado a los datos y es válido para ambos sexos. Los valores de consistencia interna son altos 
para el inventario, dos escalas y cinco factores de fobia social, las distribuciones son asimétricas positivas, las mujeres promedian más alto que los hombres, las correlaciones con la edad son negativas y débiles, se hallan evidencias de validez convergente por las correlaciones moderadas con el SIAS y SSPS y la correlación con el manejo de la impresión es significativa, pero muy débil, con menos del $2 \%$ de varianza compartida, lo que permite atribuir la asimetría de las distribuciones al aspecto psicopatológico evaluado. Precisamente lo patológico parece tomar más peso en el SPAI que en el SIAS y SSPS.

Se sugiere replicar este modelo factorial, cuya integración es novedosa y retoma la propuesta original de Turner et al. (1989), tanto en otras muestras de habla hispana como en otros idiomas, usando casos clínicos y participantes de población general para poder explorar la utilidad de una puntuación diferencial entre la escala de ansiedad social y agorafobia.

El promedio de la escala de fobia social dividido por su número de ítems da un valor de 2 , lo que refleja desde la categorías de respuesta de los ítems que los comportamientos evaluados son poco frecuentes; asimismo, el corte de corte de 60 para la puntuación diferencial "pura" con los 109 ítems originales (Turner at al., 1989; Olivares et al., 2002) arroja 17 casos positivos $(2.4 \%)$. Por lo tanto, cualquier intervención con estos estudiantes debe tener un enfoque de casos individuales, aconsejándose terapia cognitivo-conductual con formato grupal (Antona, 2009), no estando justificada ninguna intervención colectiva.

\section{REFERENCIAS}

American Psychiatric Association (2000). Diagnostic and Statistical Manual of Mental Disorders, Fourth Edition, Text Revision (DSM-IV-TR). Washington, DC: American Psychiatric Association. doi:10.1176/appi.books.978089042334 $\underline{9}$

American Psychological Association (2002). Ethical principles of psychologists and code of conduct. American Psychologist, 57, 1060-1073. doi:10.1037//0003-066X.57.12.1060

Antona, C. (2009). Fobia social: evaluación y tratamiento. México, DF: Trillas.

Beesdo, K., Knappe, S. y Pine, D. S. (2009). Anxiety and anxiety disorders in children and adolescents: Developmental issues and implications for DSM-V. Psychiatric Clinics of North America, 32, 483-524. doi:10.1016/i.psc.2009.06.002

Bögels, S. M., Alden, L., Beidel, D. C., Clark, L. A., Pine, D., Stein, M. B. y Voncken M. (2010). Social anxiety disorder: Questions and answers for the DSM-V. Depression \& Anxiety, 27, 168-189. doi:10.1002/da.20670

Butcher, J., Dahlstrom, W., Graham, J., Tellegen, A. y Kaemer, B. (1989). Manual for the restandardized Minnesota Multiphasic Personality Inventory: MMPI-2. Minneapolis, MN: University of Minnesota Press.

Byrne, B. M. (2010). Structural equation modeling with AMOS. New York, NY: Routledge. doi:10.1207/S15327574IJT0101_4

García-Cadena, C. H. (2009). Cómo investigar en psicología. México: Trillas.

García-Lopez, L. J., Olivares, J., Hidalgo, M. D., Beidel, D. C. y Turner, S. M. (2001). Psychometric properties of the Social Phobia and Anxiety Inventory, the Social Anxiety Scale for Adolescents, the Fear of Negative Evaluation scale and the Social Avoidance and Distress scale in an adolescent Spanish sample. 
Journal of Psychopathology and Behavioral Assessment, 23, 51-59. doi:10.1023/A:1011043607878

Goldberg, D. P., Krueger, R. F., Andrews, G. y Hobbs, M. J. (2009). Emotional disorders: Cluster 4 of the proposed meta-structure for DSM-V and ICD-11. Psychological Medicine, 39, 20432059. doi:10.1017/S0033291709990298

Hofmann, S. G., Anu-Asnaani, M. A. y Hinton, D. E. (2010). Cultural aspects in social anxiety and social anxiety disorder. Depression \& Anxiety, 27, 1117-27. doi:10.1002/da.20759.

Hofmann, S. G. y DiBartolo, P. M. (2000). An instrument to assess selfstatements during public speaking: Scale development and preliminary psychometric properties. Behavior Therapy, 31, 499-515. doi:10.1016/S0005-7894(00)80027-1

Inglés, C. J., Martínez-Monteagudo, M. C., Delgado, B., Torregrosa, M. S., Redondo, J., Benavides, G., GarcíaFernández, J. M. y García-López, L. J. (2008). Prevalencia de la conducta agresiva, conducta prosocial y ansiedad social en una muestra de adolescentes españoles: un estudio comparativo. Infancia y Aprendizaje, 31, 449-461.

Inglés, C. J., Piqueras, J. A., GarcíaFernández, J. M., García-López, L. J., Delgado, B. y Ruiz-Esteban, C. (2010). Diferencias de género y edad en respuestas cognitivas, psicofisiológicas y motoras de ansiedad social en la adolescencia Psicothema, 22, 376-381.

Mattick, R. P. y Clarke, J. C. (1998). Development and validation of measures of social phobia scrutiny fear and social interaction anxiety. Behaviour Research and Therapy, 36, 455-470. doi:10.1016/S00057967(97)10031-6

Medina-Mora, M. E., Borges, G., Lara, C., Benjet, C., Blanco, J., Fleiz, C., Villatoro, J., Rojas, E., Zambrano, J., Casanova, L. y Aguilar, S. (2003).
Prevalencia de trastornos mentales y uso de servicios: resultados de la Encuesta Nacional de Epidemiología Psiquiátrica en México. Salud Mental, 26(4), 1-16.

Moral, J. (2006). Análisis factorial confirmatorio. En R. Landero y M. T. González (eds.), Estadística con SPSS y metodología de la investigación (pp. 445-528). México, DF: Trillas.

Moral, J., García, C. H. y Antona, C. J. (2012). Traducción y validación del Inventario Balanceado de Deseabilidad Social al Responder en una muestra probabilística de estudiantes universitarios mexicanos. Revista de Psicología GEPU, 3(2), 20-32.

Olivares, J., García-López, L. J., Hidalgo, M. D. y Caballo, V. E. (2004). Relationships among social anxiety measures and its invariance: $A$ Confirmatory Factor Analysis. European Journal of Psychological Assessment, 20, 172-179.

Olivares, J., García-López, L. J., Hidalgo, M. D., La Greca, A. M., Turner, S. M. y Beidel, D. C. (2002). A pilot study on normative data for two social anxiety measures: The Social Phobia and Anxiety Inventory and the Social Anxiety Scale for Adolescents. International Journal of Clinical and Health Psychology, 2, 467-476.

Olivares, J., García-López, L. J., Hidalgo, M. D., Turner, S. M. y Beidel, D. C. (1999). The Social Phobia and Anxiety Inventory: Reliability and validity in an adolescent Spanish population. Journal of Psychopathology and Behavioral Assessment, 21, 67-78.

Olivares, J., Piqueras, J. A. y Alcázar, A. I. (2006). Características sociodemográficas y psicológicas de la fobia social en adolescentes. Psicothema, 18, 207-212.

Olivares, J., Vera-Villarroel, P., RosaAlcázar, A. I., Kuhne, W., Montesinos, L. y López-Pina, J. A. (2010). The social phobia and anxiety inventory: First results of the reliability and 
structural validity in chilean adolescents. Universitas Psychologica, 9, 149-160.

Osman, A., Barrios, F. X., Haupt, D., King, K., Osman, J. R. y Slavens, S. (1996). The Social Phobia and Anxiety Inventory: Further validation in two nonclinical. Journal of Psychopathology and Behavioral Assessment, 18(1), 3547. doi:10.1007/BF02229101

Paulhus, D. L. (1998). Manual for Balanced Inventory of Desirable Responding: version 7. Toronto, ON: Multi-Health Systems.

Pauhlus, D. L. (2002). Socially desirable responding: The evolution of a construct. En H. Brau, D. Jackson y D. $\mathrm{E}$. Wiley (Ed.), The role of constructs in psychological and educational measurement (pp. 46-69). Mahwah NJ: Lawrence Erlbaum.

Piqueras, J. A., Olivares, J. y López-Pina, J. A. (2008). A new proposal for the subtypes of social phobia in a sample of Spanish adolescents. Journal of Anxiety Disorders, 22, 67-77. doi:10.1016/j.janxdis.2007.01.007

Roberson-Nay, R., Strong, D. R., Nay, W. T., Beidel, D. C. y Turner, S. M. (2007). Development of an abbreviated Social Phobia and Anxiety Inventory (SPAl) using item response theory: the SPAI23. Psychology Assessment, 19,133145. doi:10.1037/1040-3590.19.1.133

Rodríguez, M. N. y Ruíz, M. A. (2008). Atenuación de la asimetría y de la curtosis de las puntuaciones observadas mediante transformaciones de variables: Incidencia sobre la estructura factorial. Psicológica, 29(2), 205-227.

Sartori, R. (2006). The bell curve in psychological research and practice: myth or reality? Quality and Quantity, 40, 407-418. doi:10.1007/s11135-0056104-0

Stahl, S. M. (2001). Effects of estrogen on the central nervous system [Brainstorms]. Journal of Clinical \begin{tabular}{lcr}
$\begin{array}{l}\text { Psychiatry, } \\
\text { doi:10.4088/JCP.v62n0501 }\end{array}$ & 317-318 \\
\hline
\end{tabular}

Thrusfield, M., Ortega, C., de Blas, I. Noordhuizen, J. y Frankena, K. (2001). Win Episcope 2.0. Improved epidemiological software for veterinary medicine. The Veterinary Record, 148, 567-572. doi:10.1136/vr.148.18.567

Turner, S. M., Beidel, D. C., Dancu, C. V. y Stanley, M. A. (1989). An empirically derived inventory to measure social fears and anxiety: the social phobia and anxiety inventory. Psychological Assesment, $\quad 1, \quad 35-40$. doi:10.1037/1040-3590.1.1.35

Young, F. W. (2010). ViSta "The Visual Statistics System", version 7.9.2.5 [computer software] [on-line]. Disponible en: URL: http://www.visualstats.org

Yuan, K. H. y Bentler, P. M. (2004). On chisquare difference and z-tests in mean and covariance structure analysis when the base model is misspecified. Educational and Psychological Measurement, 64, 737-757. doi:10.1177/0013164404264853

Wolitzky-Taylor, K., Castriotta, N., Lenze, E., Stanley, M. y Craske, M. G. (2010). Anxiety disorders in late age: A comprehensive review. Depression \& Anxiety, 27, 190-211. doi: 10.1002/da.20653.

Zhou, X., Xu, Q., Inglés, C. J., Hidalgo, M. D. y La Greca, A. M. (2008). Reliability and validity of the Chinese version of the Social Anxiety Scale for Adolescents. Child Psychiatry and Human Development, 39, 185-200. doi:10.1007/s10578-007-0079-0 
ANEXO 1.

Basándose en su experiencia personal, por favor diga qué tan frecuentemente experimenta estos sentimientos y pensamientos en situaciones sociales. Por favor, utilice la escala de abajo y encierre el número que mejor refleje qué tan frecuentemente experimenta usted estas respuestas.

\begin{tabular}{|c|c|c|c|c|c|c|}
\hline $\mathbf{1}$ & $\mathbf{2}$ & $\mathbf{3}$ & $\mathbf{4}$ & $\mathbf{5}$ & $\mathbf{6}$ & $\mathbf{7}$ \\
\hline Nunca & $\begin{array}{c}\text { Con muy } \\
\text { poca } \\
\text { frecuencia }\end{array}$ & $\begin{array}{c}\text { Con poca } \\
\text { frecuencia }\end{array}$ & $\begin{array}{c}\text { Con } \\
\text { frecuencia }\end{array}$ & $\begin{array}{c}\text { Con } \\
\text { bastante } \\
\text { frecuencia }\end{array}$ & $\begin{array}{c}\text { Con mucha } \\
\text { frecuencia }\end{array}$ & Siempre \\
\hline
\end{tabular}

(Las 7 columnas de respuestas de frecuencia se han suprimido para ahorrar espacio. Con el número entre paréntesis y el ítem en cursiva se señalan los que quedaron en el modelo con mejor ajuste, asimismo se identifica al factor que pertenece: $A G=$ Escala de agorafobia y FS = Escala de fobia social: $A R I=$ Factor de ansiedad en relaciones interpersonales con personas del sexo opuesto y personas en general, TAE = Factor de timidez ante autoridades y extraños, $C B P=$ Factor de comer y beber en público, EVA = Factor de evitación/abandono de situaciones sociales generadoras de ansiedad, y MRF = Factor de miedos y reacciones fisiológicas).

1. Me siento nervioso cuando entro en contacto con situaciones sociales donde hay un grupo pequeño.

2. Me siento nervioso cuando entro en contacto con situaciones sociales donde hay un grupo grande.

3. Me siento nervioso cuando estoy en una situación social y me convierto en el centro de atención.

4. Me siento nervioso cuando estoy en una situación social y se espera que realice alguna actividad.

5. Me siento nervioso cuando hablo frente a una audiencia.

6. Me siento nervioso cuando hablo en una pequeña reunión informal.

7. Me siento tan nervioso de asistir a reuniones sociales que evito estas situaciones.

8. Me siento tan nervioso en situaciones sociales que abandono las reuniones.

(9). Me siento nervioso estando en una reunión pequeña con:
a. Extraños
b. Figuras de autoridad
(c) Sexo opuesto [FS_ARI]
(d) Personas en general. [FS_ARI]

(10). Me siento nervioso estando en una reunión grande con:
a. Extraños
b. Figuras de autoridad
(c) Sexo opuesto [FS_ARI]
d. Personas en general.

11. Me siento nervioso estando en un bar o restaurante con:
a. Extraños
b. Figuras de autoridad
c. Sexo opuesto
d. Personas en general.

(12). Me siento nervioso y no sé qué hacer estando en una nueva situación con:
a. Extraños
b. Figuras de autoridad
(c) Sexo opuesto [FS_ARI]
(d) Personas en general. [FS_ARI]

(13). Me siento nervioso y no sé qué hacer estando en una situación que implique confrontación con:
a. Extraños
b. Figuras de autoridad
(c) Sexo opuesto [FS_ARI]
(d) Personas en general. [FS_ARI]

14. Me siento nervioso y no sé qué hacer estando en una situación vergonzosa con:
a. Extraños
b. Figuras de autoridad 

c. Sexo opuesto
d. Personas en general.

15. Me siento nervioso al hablar de sentimientos íntimos con:
a. Extraños
b. Figuras de autoridad
c. Sexo opuesto
d. Personas en general.

(16). Me siento nervioso cuando doy una opinión a:
a. Extraños
b. Figuras de autoridad
(c) Sexo opuesto [FS_ARI]
d. Personas en general.

17. Me siento nervioso hablando de negocios con:
a. Extraños
b. Figuras de autoridad
c. Sexo opuesto
d. Personas en general.

(18). Me siento nervioso cuando me acerco y/o inicio una conversación con:
(a) Extraños [FS_TAE]
(b) Figuras de autoridad [FS_TAE]
c. Sexo opuesto
d. Personas en general.

(19). Me siento nervioso cuando tengo que interactuar por más de unos cuantos minutos con:
(a) Extraños [FS_TAE]
(b) Figuras de autoridad [FS_TAE]
c. Sexo opuesto
d. Personas en general.

20. Me siento nervioso cuando tomo (cualquier bebida) o ingiero alimentos delante de:
a. Extraños
b. Figuras de autoridad
c. Sexo opuesto
d. Personas en general.

(21). Me siento nervioso al escribir o teclear delante de:
(a) Extraños [FS_CBP]
(b) Figuras de autoridad [FS_CBP]
(c) Sexo opuesto [FS_CBP]
(d) Personas en general. [FS_CBP]

22. Me siento nervioso cuando hablo frente a:
a. Extraños
b. Figuras de autoridad
c. Sexo opuesto
d. Personas en general.

23. Me siento nervioso cuando soy criticado o rechazado por:
a. Extraños
b. Figuras de autoridad
c. Sexo opuesto
d. Personas en general.

(24). Intento evitar situaciones sociales en donde hay:
(a) Extraños [FS_EVA]
(b) Figuras de autoridad [FS_EVA]
(c) Sexo opuesto [FS_EVA]
(d) Personas en general [FS_EVA].

(25). Abandono las situaciones sociales en donde haya:
(a) Extraños [FS_EVA]
(b) Figuras de autoridad [FS_EVA] 

(c) Sexo opuesto [FS_EVA]
(d) Personas en general [FS_EVA].

(26). Antes de entrar en contacto con una situación social pienso en todas las cosas que pueden salir mal. Los tipos de pensamiento que tengo son:
a. ¿Estaré vestido apropiadamente?
(b) Probablemente cometeré un error y pareceré un tonto [FS_MRF]
(c) ¿Qué haré si nadie me habla? [FS_MRF]
(d) Si hay un silencio en la conversación ¿sobre qué puedo hablar? [FS_MRF]
(e) La gente se dará cuenta de lo nervioso que estoy. [FS_MRF]

27. Me siento nervioso antes de entrar en contacto con una situación social.

28. Se me va la voz o se me quiebra cuando estoy hablando en una situación social.

29. Es poco probable que le hable a la gente hasta que ellos me hablen primero.

(30). Tengo pensamientos problemáticos cuando estoy en una situación social. Por ejemplo:
a. Desearía poder irme y evitar toda la situación
(b) Si lo estropeo de nuevo realmente perderé la confianza en mí mismo [FS_MRF]
(c) ¿Qué clase de impresión estoy dando a los demás? [FS_MRF]
d. Cualquier cosa que diga probablemente sonará estúpida.

31. Experimento lo siguiente antes de entrar en contacto con una situación social:
a. Sudoración
b. Ganas frecuentes de orinar
c. Palpitaciones cardiacas.

32. Experimento lo siguiente en una situación social:
a. Sudoración
b. Sonrojarse o ruborizarse
c. Temblores
d. Ganas frecuentes de orinar
e. Palpitaciones cardiacas.

(33). Me siento nervioso cuando estoy solo en casa. [AGO]

34. Me siento nervioso cuando estoy en un lugar extraño.

(35). Me siento nervioso cuando estoy en cualquier medio de transporte público (por ejemplo, camión, tren, avión, etc). [AGO]

(36). Me siento nervioso cuando cruzo la calle. [AGO]

(37). Me siento nervioso cuando estoy en lugares públicos llenos de gente (por ejemplo, tiendas, iglesia, cines, restaurantes, etc). [AGO]

(38). Estar en grandes espacios abiertos me hace sentir nervioso. [AGO]

(39). Me siento nervioso cuando estoy en lugares cerrados (elevadores, túneles, etc). [AGO]

(40). Estar en lugares elevados me hace sentir nervioso (por ejemplo, edificios altos). [AGO]

(41). Me siento nervioso cuando estoy esperando en una fila larga. [AGO]

(42). Hay veces en las que siento que tengo que agarrarme de las cosas porque temo caerme. [AGO]

43. Cuando salgo de la casa y voy a lugares públicos voy acompañado de un familiar o amigo.

(44). Me siento nervioso cuando viajo en carro. [AGO]

(45). Hay ciertos lugares a los que no voy porque podría sentirme atrapado. [AGO] 